\title{
Intérêt de l'analyse élémentaire des produits viticoles : dosage de vingt-cinq éléments par spectrométrie d'émission atomique dans un plasma à couplage induit*
}

\author{
J.-B. Fournier ${ }^{1}$, O. Hirsch ${ }^{2}$ et G.J. Martin ${ }^{1}$ \\ ${ }^{1}$ Laboratoire d'électrochimie et d'analyses isotopiques des métabolismes, CNRS UPRES-A Q6006, \\ Université de Nantes, 2 chemin de la Houssinière, 44300 Nantes, France \\ ${ }^{2}$ Laboratoire d'application Jobin-Yvon, 16 rue du Canal, 91160 Longjumeau, France
}

\begin{abstract}
It is well known that at least fifteen elements have a physiological effect on the growth of the wine. Some of these elements have a chemical effect during the fermentation. In addition to the previous elements, nine minor elements can be analyzed in wine for toxic levels. This paper underlines the particular behavior of some elements and their importance concerning the viticulture products and describes a method of analyzing twenty-five elements.
\end{abstract}

Key words. trace elements - wine physiology - toxicity, authentication - ICP-AES.

\section{Introduction}

Les progrès effectués tant en agronomie qu'en chimie analytique et l'importance qui est désormais accordée à l'envi- ronnement amènent à s'interroger sur les moyens à mettre en œuvre pour contrôler la culture et la transformation des produits viticoles. La chimie analytique en particulier a permis de mettre au point des techniques de contrôle fiables et 


\section{Original articles}

de limiter ainsi les fraudes et les abus. La prise en compte de la protection de l'environnement et de la toxicologie des produits chimiques ont conduit l'industrie agro-alimentaire à établir des normes strictes concernant les teneurs de certains constituants. L'analyse des éléments traces s'inscrit en bonne place pour asseoir la législation en offrant les possibilités de contrôler l'application des règlements et des normes de fabrication.

Mais l'analyse des éléments traces peut également être un soutien pour les producteurs eux-mêmes, tout d'abord en leur permettant de caractériser leur cru et de pouvoir l'authentifier, mais aussi en leur donnant les moyens de contrôler certaines étapes de la fermentation et d'évaluer avec précision les risques liés aux casses métalliques pour ajuster certains traitements.

Les éléments traces sont absorbés par la plante sous forme libre ou sous forme chélatée. Deux modes d'absorption sont mis en œuvre : le mode actif qui nécessite un apport d'énergie métabolique pour pouvoir s'opérer et le mode passif qui s'effectue par diffusion à travers les tissus. Le mode actif concerne principalement les éléments physiologiquement indispensables à la vigne, le mode passif concerne les autres éléments, dont certains peuvent provoquer des réactions toxiques pour la plante ou présenter un risque de toxicité pour l'homme. Les traitements phyto-sanitaires peuvent être responsables de l'apport des éléments traces, le cas le plus courant étant celui du traitement cuprique. Enfin la présence de certains éléments comme le plomb semble trouver son origine dans la pollution atmosphérique. Les exemples cités dans le paragraphe suivant témoignent de l'importance des éléments minéraux pour la viticulture. Chaque élément jouant un rôle physiologique peut être responsable de carences graves nuisant au développement de la plante, l'analyse du moût ou du vin peut permettre de déceler ces carences. De très nombreux éléments s'avèrent toxiques à fortes concentrations, des teneurs anormalement élevées doivent être interprétées.

Un des rôles du potassium est de neutraliser les charges négatives des acides organiques et des protéines et de participer au potentiel osmotique cellulaire. Il permet la migration de certains métabolites à travers les parois cellulaires, et est également cofacteur de nombreuses réactions. Le calcium et le magnésium catalysent certaines réactions enzymatiques et entrent dans la composition des parois des cellules pour neutraliser les fonctions acides des composés pectiques. Le soufre forme les fonctions thiols des acides aminés. Le fer est indispensable car il joue un rôle dans les réactions de la respiration cellulaire et dans la synthèse chlorophyllienne. Le molybdène entre dans la constitution des réductases et le cuivre dans celle des oxydases. Le manganèse catalyse des réactions enzymatiques. Le bore permet la migration des sucres à travers la plante. Le zinc enfin, est un constituant de l'anhydrase carbonique.

D'autre part, la connaissance des teneurs en éléments traces du moût de raisin permet de prévenir certains accidents durant la vinification [2]. Plusieurs phénomènes de précipitation de complexes métalliques peuvent être la cause de vins troubles. Des teneurs élevées en potassium et calcium, associées à une forte concentration en acide tartrique entraînent l'apparition de cristaux de tartre sous forme de tartrate de potassium et de tartrate de calcium; en effet, la solubilité des ions tartrate diminue avec l'augmentation du degré alcoolique durant la fermentation. L'oxydation des ions $\mathrm{Fe}^{2+}$ en ions $\mathrm{Fe}^{3+}$ peut entraîner la casse blanche par réaction avec l'acide phosphorique ou la casse bleue par précipitation avec les tanins. Enfin le cuivre en se fixant sur les levures peut inhiber leur action, le risque le plus grave est la casse cuivrique qui apparaît dans les vins blancs et rosés suite à la précipitation de sulfure de cuivre.

Les éléments traces constituent assurément des traceurs fiables pour reconnaître l'origine d'un vin. Les techniques statistiques multidimensionnelles telles que l'analyse linéaire discriminante [3] ont permis de caractériser l'origine des vins et au besoin d'authentifier certains crus. De nombreux auteurs ont développé des recherches dans ce domaine, Herrero-Latorre [4] a caractérisé les vins de Galice et Gonzales-Larraina [5] a étudié les vins rouges de la région Rioja.

L'utilisation simultanée des isotopes stables et des éléments traces a permis à Day et Martin [6] de différencier les principales zones de production françaises, et des méthodes similaires ont été appliquées sur les vins américains par Kwan [7]. Une étude de Seeber [8] montre également la possibilité de caractériser les différents millésimes d'une même production. Enfin, de nombreux auteurs ont montré que des corrélations existent entre la teneur d'un élément dans les vins et dans les sols, les éléments traces peuvent ainsi contribuer à la définition des terroirs. Une étude de Stobbaerts [9] sur le manganèse démontre que la teneur de cet élément dans le vin dépend beaucoup du sol de culture.

En ce qui concerne les contaminants, c'est-à-dire les éléments qui apportent des risques de toxicité, bien que présents à de très faibles concentrations dans le vin, ils doivent impérativement être surveillés car ils constituent un risque majeur pour l'alimentation humaine. Teissèdre [10] a étudié les variations des teneurs en cadmium au cours de l'élaboration des vins de Côtes du Rhône. Pertoldi-Marletta [11] a montré l'influence de la pollution atmosphérique à travers la proximité d'une route sur les concentrations en chrome et en nickel. Les récentes études de Augagneur [12] montrent que l'essentiel du plomb contenu dans les vins provient de la pollution par les gaz d'échappement. Cabrera-Vique [13] ou de la Torre [14] se sont intéressées à ce que représente l'apport du vin par rapport à l'ingestion journalière de métaux lourds. Il importe donc de contrôler les teneurs de ces éléments dans le vin [15], car elles constituent des données importantes pour évaluer l'influence de la pollution sur la production agricole et par là même dans notre alimentation. L'analyse des éléments traces dans les produits viticoles constitue une aide pour le producteur afin de surveiller l'élaboration de son vin. Mais c'est aussi un moyen d'authentifier un produit ou d'évaluer certains risques de toxicité.

\section{Matériel et méthodes}

\section{Appareillage}

$\mathrm{Vu}$ le grand nombre d'éléments concernés, la spectrométrie d'émission atomique dans un plasma à couplage induit (ICPAES) [16] est une technique de choix permettant d'allier la fiabilité des résultats et la rapidité d'analyse. La méthode décrite dans ce travail a été développée sur l'appareil JobinYvon Panorama pour l'analyse simultanée de vingt-quatre 
éléments. L'analyse du rubidium a été effectuée en mode séquentiel sur l'appareil Jobin-Yvon JY 2000.

\section{Préparation des échantillons}

La présence d'alcool en abaissant la température du plasma perturbe l'analyse, l'utilisation d'un nébuliseur thermostaté à $0{ }^{\circ} \mathrm{C}$ associée à la réduction du débit du gaz auxiliaire permet de confiner les éléments dans la torche et résout ainsi le problème posé par l'alcool lors de l'analyse. Concernant l'analyse des moûts et des lies durant la fermentation, la présence de différentes phases dans les soutirages effectués ont conduit à minéraliser les échantillons. Une prise d'essai de cinq grammes d'échantillons est minéralisée, en présence de $2 \mathrm{ml}$ d'acide chlorhydrique et de $0,5 \mathrm{ml}$ d'acide nitrique de puretés analytiques, au moyen du digesteur Prolabo A-300 qui utilise le chauffage par micro-ondes focalisées en condition de reflux total. Le minéralisat obtenu est filtré et le volume est ajusté à $25 \mathrm{ml}$ avec de l'eau déminéralisée.

\section{Gamme d'étalonnage}

Le potassium qui est l'élément majeur et qui se trouve dans le vin à une concentration voisine $\mathrm{du} \mathrm{g} / \mathrm{l}$ peut comme tous les alcalins avoir un effet inhibiteur sur l'émission des autres éléments. Le minéralisat analysé correspondant à une dilution au cinquième de l'échantillon, $200 \mathrm{mg} / \mathrm{l}$ de potassium ont été introduits dans tous les standards réalisés qui ont également été acidifiés. Les tableaux I et II résument pour chaque élément dosé la longueur d'onde de travail, l'étendue de la gamme de calibration utilisée ainsi que les paramètres de la régression linéaire. Les résultats obtenus et la comparaison avec des solutions réelles dopées par ajouts dosés montrent que l'utilisation de solutions de référence synthétiques pour la calibration est fiable, excepté pour l'arsenic, le sélénium et le rubidium. Pour ces trois éléments, les valeurs d'étalonnage ont été obtenues par ajouts dosés.

Tableau I. Eléments majeurs, paramètres analytiques.

\begin{tabular}{lccccc}
\hline Élément & $\begin{array}{c}\text { Longueur } \\
\text { d'onde } \\
(\mathrm{nm})\end{array}$ & $\begin{array}{c}\text { Gamme } \\
\text { de calibration } \\
(\mathrm{mg} / \mathrm{l})\end{array}$ & $\begin{array}{c}\text { Pente de la droite } \\
\text { de régression } \\
(\mathrm{n=10})\end{array}$ & $\begin{array}{c}\text { \% répétabilité } \\
\text { sur la pente } \\
(n=10)\end{array}$ & $\begin{array}{c}\text { Coefficient } \\
\text { de régression } \\
r^{2}\end{array}$ \\
\hline $\mathrm{Ca}$ & 317,933 & $0-50$ & 1,62 & 6,7 & 0,99927 \\
$\mathrm{~K}$ & 766,490 & $0-500$ & 6,24 & 11,5 & 0,99925 \\
$\mathrm{Mg}$ & 279,079 & $0-5$ & 121,93 & 6,4 & 0,99913 \\
$\mathrm{Na}$ & 589,592 & $0-10$ & 0,33 & 15,4 & 0,99823 \\
$\mathrm{P}$ & 178,225 & $0-50$ & 18,09 & 6,2 & 0,99975 \\
$\mathrm{~S}$ & 180,672 & $0-50$ & 22,26 & 7,0 & 0,99994 \\
\hline
\end{tabular}

Tableau II. Eléments mineurs, paramètres analytiques.

\begin{tabular}{lccccc}
\hline Élément & $\begin{array}{c}\text { Longueur } \\
\text { d'onde } \\
(\text { nm })\end{array}$ & $\begin{array}{c}\text { Gamme } \\
\text { de calibration } \\
(\mu g / l)\end{array}$ & $\begin{array}{c}\text { Pente de la droite } \\
\text { de régression } \\
(n=10)\end{array}$ & $\begin{array}{c}\text { \% répétabilité } \\
\text { sur la pente } \\
(n=10)\end{array}$ & $\begin{array}{c}\text { Coefficient } \\
\text { de régression }\end{array}$ \\
\hline $\mathrm{Ag}$ & 328,068 & $0-500$ & 0,61 & 13,7 & $r^{2}$ \\
$\mathrm{Al}$ & 396,152 & $0-500$ & 0,66 & 12,5 & 0,99994 \\
$\mathrm{As}$ & 193,695 & $0-500$ & 0,0239 & 9,8 & 0,99994 \\
$\mathrm{~B}$ & 208,959 & $0-500$ & 0,0531 & 9,9 & 0,99979 \\
$\mathrm{Ba}$ & 233,527 & $0-500$ & 0,67 & 7,1 & 1,00000 \\
$\mathrm{Cd}$ & 226,502 & $0-500$ & 0,53 & 11,3 & 0,99988 \\
$\mathrm{Cr}$ & 267,716 & $0-500$ & 0,19 & 7,9 & 0,99989 \\
$\mathrm{Cu}$ & 324,754 & $0-500$ & 0,27 & 14,2 & 0,99998 \\
$\mathrm{Fe}$ & 259,940 & $0-500$ & 0,0083 & 6,2 & 0,99998 \\
$\mathrm{Hg}$ & 184,887 & $0-100$ & 0,0827 & 2,5 & 1,00000 \\
$\mathrm{Mn}$ & 257,610 & $0-500$ & 0,93 & 5,6 & 0,99995 \\
$\mathrm{Ni}$ & 231,604 & $0-500$ & 0,15 & 7,9 & 0,99995 \\
$\mathrm{~Pb}$ & 220,353 & $0-500$ & 0,0130 & 9,8 & 0,99995 \\
$\mathrm{Rb}$ & 783,023 & $0-500$ & 0,0305 & 6,2 & 0,99966 \\
$\mathrm{Sb}$ & 206,833 & $0-500$ & 0,0091 & 7,9 & 0,99972 \\
$\mathrm{Se}$ & 196,026 & $0-500$ & 0,0076 & 9,5 & 0,99968 \\
$\mathrm{Sn}$ & 189,926 & $0-100$ & 0,0038 & 8,9 & 1,00000 \\
$\mathrm{Sr}$ & 407,771 & $0-500$ & 2,40 & 14,5 & 0,99999 \\
$\mathrm{Zn}$ & 213,856 & $0-500$ & 1,16 & 5,0 & 0,99982 \\
\hline & & & & &
\end{tabular}




\section{Résultats}

\section{Répétabilité, limites de détection et de quantification}

Les tableaux III et IV donnent la moyenne et l'écart-type calculés à partir de dix-huit mesures d'un échantillon de vin témoin. La répétabilité obtenue est satisfaisante. Les limites de détection et de quantification récapitulées dans les tableaux III et IV ont été calculées à partir de dix mesures d'étalonnage par les formules suivantes, dans lesquelles $B_{0}$ et $S D_{0}$ désignent la valeur moyenne et l'écart-type calculés sur les mesures d'un blanc analytique.

$$
L_{\text {det }}=\left(B_{0}+3 S D_{0}\right) \quad L_{\text {quan }}=\left(B_{0}+10 S D_{0}\right) .
$$

Toutes les limites de détection calculées montrent qu'il est possible d'analyser correctement les éléments majeurs et mineurs présents dans le vin. Nos méthodes permettent de contrôler les métaux lourds relativement aux normes en vigueur, et de répondre à une évolution éventuelle de ces normes.

\section{Evaluation de la fiabilité de la méthode}

Un échantillon témoin a été préparé ; cet échantillon a fait l'objet de deux ajouts à des concentrations différentes. Ces ajouts ont été effectués à partir de solutions concentrées pour ne pas modifier la matrice de l'échantillon par dilution. Les

Tableau III. Répétabilité et limites de détection, éléments majeur.

\begin{tabular}{lcccc}
\hline Élément & $\begin{array}{c}\text { Échantillon } X \\
\text { Moyenne } \\
(m g / l)(n=18)\end{array}$ & $\begin{array}{c}\text { Écart-type } \\
\text { relatif }(\%) \\
(n=18)\end{array}$ & $\begin{array}{c}\text { Limite de } \\
\text { détection } \\
m g / l\end{array}$ & $\begin{array}{c}\text { Limite de } \\
\text { quantification } \\
m g / l\end{array}$ \\
\hline $\mathrm{B}$ & 5,14 & 2,23 & 0,03 & 0,08 \\
$\mathrm{Ca}$ & 89,0 & 0,44 & 0,02 & 0,06 \\
$\mathrm{Fe}$ & 1,52 & 0,71 & 0,01 & 0,02 \\
$\mathrm{~K}$ & 928 & 3,57 & 0,42 & 1,62 \\
$\mathrm{Mg}$ & 53,7 & 4,66 & 0,03 & 0,19 \\
$\mathrm{Na}$ & 2,81 & 2,86 & 0,03 & 0,07 \\
$\mathrm{P}$ & 169 & 4,89 & 0,05 & 0,12 \\
$\mathrm{~S}$ & 90,7 & 1,58 & 0,12 & 0,26 \\
\hline
\end{tabular}

tableaux V et VI résument les résultats obtenus. Les taux de recouvrement des éléments dosés sont tous supérieurs à $90 \%$, à l'exception de l'aluminium pour lequel le taux est voisin de $85 \%$. Ces tests démontrent la fiabilité des résultats obtenus et permettent d'affirmer que les effets de matrice et les interactions possibles entre éléments ont été maîtrisés.

\section{Conclusion}

Le développement de telles méthodes dans les laboratoires permettra la constitution à l'échelon européen et mondial d'une banque de données des teneurs en éléments traces contenus dans les vins. A court ou moyen terme, une telle banque de données deviendra un outil indispensable à la caractérisation et à l'authentification des différentes zones de production, ceci dans le souci de préserver les intérêts du consommateur et la qualité des grands crus. Il faut espérer

Tableau IV. Répétabilité et limites de détection, éléments mineurs.

\begin{tabular}{lcccc}
\hline Élément & $\begin{array}{c}\text { Échantillon } X \\
\text { Moyenne } \\
(m g / l)(n=18)\end{array}$ & $\begin{array}{c}\text { Écart-type } \\
\text { relatif }(\%) \\
(n=18)\end{array}$ & $\begin{array}{c}\text { Limite de } \\
\text { détection } \\
\mu g / l\end{array}$ & $\begin{array}{c}\text { Limite de } \\
\text { quantification } \\
\mu g / l\end{array}$ \\
\hline $\mathrm{Ag}$ & $<2,7$ & & 2,7 & 7,6 \\
$\mathrm{Al}$ & 405 & 4,12 & 26 & 78 \\
$\mathrm{As}$ & $<10$ & & 10 & 34 \\
$\mathrm{Ba}$ & 230 & 2,98 & 1,4 & 5,8 \\
$\mathrm{Cd}$ & 11,6 & 4,05 & 0,7 & 1,5 \\
$\mathrm{Cr}$ & 23,0 & 5,53 & 0,6 & 0,8 \\
$\mathrm{Cu}$ & 78 & 4,61 & 6 & 10 \\
$\mathrm{Hg}$ & $<3$ & & 3 & 22 \\
$\mathrm{Mn}$ & 339 & 1,74 & 0,4 & 1,2 \\
$\mathrm{Ni}$ & 28,1 & 6,07 & 0,7 & 2,6 \\
$\mathrm{~Pb}$ & $<1,4$ & & 1,4 & 4,0 \\
$\mathrm{Rb}$ & 771 & 1,11 & 9 & 18 \\
$\mathrm{Sb}$ & $<6$ & & 6 & 20 \\
$\mathrm{Se}$ & $<11$ & & 11 & 39 \\
$\mathrm{Sn}$ & $<5$ & & 5 & 16 \\
$\mathrm{Sr}$ & 167 & 2,37 & 1,9 & 5,7 \\
$\mathrm{Zn}$ & 97 & 2,60 & 2,4 & 6,1 \\
\hline
\end{tabular}

Tableau V. Recouvrement de concentrations ajoutées, éléments majeurs.

\begin{tabular}{lccccc}
\hline Élément & $\begin{array}{c}\text { Témoin } \\
\text { sans ajout } \\
(m g / l)\end{array}$ & $\begin{array}{c}\text { Ajout } n^{\circ} 1 \\
\text { valeur } \\
(m g / l)\end{array}$ & $\begin{array}{c}\text { Ajout } n^{\circ} 2 \\
\text { valeur } \\
(m g / l)\end{array}$ & $\begin{array}{c}\text { Ajout } n^{\circ} 1 \\
\text { Taux de } \\
\text { recouvrement }\end{array}$ & $\begin{array}{c}\text { Ajout } n^{\circ} 2 \\
\text { Taux de } \\
\text { recouvrement }\end{array}$ \\
\hline $\mathrm{B}$ & 0,89 & 0,5 & 1 & $97 \%$ & $90 \%$ \\
$\mathrm{Ca}$ & 33,7 & 5 & 10 & $110 \%$ & $98 \%$ \\
$\mathrm{Fe}$ & 0,79 & 0,5 & 1 & $100 \%$ & $95 \%$ \\
$\mathrm{~K}$ & 281 & 50 & 100 & $91 \%$ & $93 \%$ \\
$\mathrm{Mg}$ & 16,8 & 5 & 10 & $95 \%$ & $96 \%$ \\
$\mathrm{Na}$ & 2,1 & 5 & 10 & $90 \%$ & $103 \%$ \\
$\mathrm{P}$ & 45,0 & 5 & 10 & $93 \%$ & $100 \%$ \\
$\mathrm{~S}$ & 24,3 & 5 & 10 & $96 \%$ & $98 \%$ \\
\hline
\end{tabular}




\section{Original articles}

Tableau VI. Recouvrement de concentrations ajoutées, éléments mineurs.

\begin{tabular}{lccccc}
\hline Élément & $\begin{array}{c}\text { Témoin } \\
\text { sans ajout } \\
(\mu g / l)\end{array}$ & $\begin{array}{c}\text { Dopage } n^{\circ} \\
\text { valeur } \\
(\mu g / l)\end{array}$ & $\begin{array}{c}\text { Dopage } n^{\circ} 2 \\
\text { valeur } \\
(\mu g / l)\end{array}$ & $\begin{array}{c}\text { Dopage } n^{\circ} 1 \\
\text { Taux de } \\
\text { recouvrement }\end{array}$ & $\begin{array}{c}\text { Dopage } n^{\circ} 2 \\
\text { Taux de } \\
\text { recouvrement }\end{array}$ \\
\hline $\mathrm{Ag}$ & $<2,7$ & 500 & 1000 & $91 \%$ & $89 \%$ \\
$\mathrm{Al}$ & 112 & 500 & 1000 & $86 \%$ & $84 \%$ \\
$\mathrm{As}$ & $<10$ & 500 & 1000 & $101 \%$ & $101 \%$ \\
$\mathrm{Ba}$ & 43 & 500 & 1000 & $95 \%$ & $91 \%$ \\
$\mathrm{Cd}$ & $<0,7$ & 500 & 1000 & $99 \%$ & $94 \%$ \\
$\mathrm{Cr}$ & 11 & 500 & 1000 & $105 \%$ & $101 \%$ \\
$\mathrm{Cu}$ & 137 & 500 & 1000 & $96 \%$ & $95 \%$ \\
$\mathrm{Hg}$ & $<3$ & 500 & 1000 & $104 \%$ & $96 \%$ \\
$\mathrm{Mn}$ & 280 & 500 & 1000 & $100 \%$ & $96 \%$ \\
$\mathrm{Ni}$ & 22 & 500 & 1000 & $98 \%$ & $93 \%$ \\
$\mathrm{~Pb}$ & $<1,4$ & 500 & 1000 & $94 \%$ & $89 \%$ \\
$\mathrm{Rb}$ & 157 & 500 & 1000 & $101 \%$ & $94 \%$ \\
$\mathrm{Sb}$ & $<6$ & 500 & 1000 & $99 \%$ & $96 \%$ \\
$\mathrm{Se}$ & $<11$ & 500 & 1000 & $104 \%$ & $101 \%$ \\
$\mathrm{Sn}$ & $<5$ & 500 & 1000 & $107 \%$ & $101 \%$ \\
$\mathrm{Sr}$ & 54 & 500 & 1000 & $95 \%$ & $92 \%$ \\
$\mathrm{Zn}$ & 278 & 500 & 1000 & $92 \%$ & $87 \%$ \\
\hline
\end{tabular}

que les chercheurs, les producteurs et les différents intervenants sauront coopérer sous l'égide de la communauté européenne pour parvenir à édifier cette banque de données. L'exemple de la banque de données contenant les rapports isotopiques stables, dressée pour étudier et détecter la chaptalisation des vins, démontre clairement l'utilité et la faisabilité de tels projets.

\section{Références}

1. Champagnol, F. Eléments de physiologie de la vigne, edité par l'auteur, ISBN 2-9500614-0-0, 1984.

2. Peynaud, E. Connaissance et travail du vin, DUNOD, Paris, 1975.

3. Symonds, P.; Cantagrel, R. Ann. Fais. Exp. Chim. 1982, 75, 805, 63-74.

4. Herrero-Latorre, C.; Medina, B. J. Inter. Sci. Vigne Vin 1990, 24(4), 147-156.

5. Gonzales-Larraina, M.; Gonzales, A.; Medina, B. Conn. Vigne Vin 1987, 21(2) 127-140.
6. Day, M.P.; Zhang, B.; Martin, G. J. J. Sci. Food Agric. 1995, 67, 113-123.

7. Kwan, W.; Kowalski, B. R.; Skogerboe, R. K. J. Agric. Food Chem. 1979, 27(6) 1321-1325.

8. Seeber, R.; Sferlazzo, G.; Leardi, R.; Dalla Serra, A.; Versini, G. J. Agric. Food Chem. 1991, 39, 1764-1769.

9. Stobbaerts, R.; Robberecht, H.; Haesen, F.; Deelstra, H. Inter. J. Vit. Nutr. Res. 1994, 64, 233-236.

10. Teissèdre, P.L.; Cabanis, M.T.; Daumas, F.; Cabanis, J.C. Sci. Alim. 1994, 14, 741-749.

11. Pertoldi-Marletta, G.; Gabrielli-Favretto, L.; Favretto L. Food Add. Cont. 1989, 6(2) 219-225.

12. Augagneur, S. Actes du Symposium In Vino Analytica Scientia, Bordeaux 1997; pp 204-207.

13. Cabrera-Vique, C.; Teissèdre, P.L.; Cabanis, M.T.; Cabanis, J.C. J. Agric. Food Chem. 1997, 45, 1808-1811.

14. de la Torre, C.; Analusis Magazine 1997, 25(3) M21-M26.

15. Scollary, G. R. Analusis Magazine 1997, 25(3) M26-M30.

16. Fournier, J. B.; Hirsch, O. Actes du Symposium In Vino Analytica Scientia, Bordeaux 1997; pp 532-535. 